\title{
Novel mutations of the SERPINF1 and FKBP10 genes in Chinese families with autosomal recessive osteogenesis imperfecta
}

\author{
HAO ZHANG, YANG XU, HUA YUE, CHUN WANG, JIEMEI GU, JINWEI HE, \\ WENZHEN FU, WEIWEI HU and ZHENLIN ZHANG \\ Department of Osteoporosis and Bone Diseases, Metabolic Bone Diseases and Genetic Research, \\ Shanghai Key Clinical Centre for Metabolic Diseases, Shanghai Jiao Tong University \\ Affiliated Sixth People's Hospital, Shanghai 200233, P.R. China
}

Received September 17, 2017; Accepted March 1, 2018

DOI: $10.3892 /$ ijmm.2018.3542

\begin{abstract}
The aim of the present study was to characterize the clinical manifestations and identify the mutations of Serpin family F member 1 (SERPINF1) and FK506 binding protein 10 (FKBP10) genes in Chinese patients with osteogenesis imperfecta (OI). Using whole-exome sequencing in the first and third probands, a novel mutation was identified in SERPINF1 and a novel compound heterozygous mutation was revealed in FKBP10. Using Sanger sequencing, an additional novel mutation in SERPINF1 was identified in a proband of family 2 . In family 1 , the proband presented with a novel homozygous missense mutation of the SERPINF1 gene, c.1067T >A (V356E). In family 2, the proband had a novel homozygous deletion mutation of the SERPINF1 gene, c.283+473_643+104del (p.Ala96_Gly215del). Serum pigment-epithelium-derived factor concentration was not detected in probands with OI type VI. For both families, the proband's father was demonstrated to have a heterozygous mutation of SERPINF1, whereas no mutations was detected in the probands' mothers. An assessment of allelic copy numbers revealed a deletion of SERPINF1 in the mother of family 1 . The results of the present study demonstrate that patients may have mild symptoms of OI with a large fragment deletion in the SERPINF1 gene. Thus, the phenotype of Chinese patients with type VI OI is milder than that of Caucasian and Korean patients. In family 3, the
\end{abstract}

Correspondence to: Professor Zhenlin Zhang, Department of Osteoporosis and Bone Diseases, Metabolic Bone Diseases and Genetic Research, Shanghai Key Clinical Centre for Metabolic Diseases, Shanghai Jiao Tong University Affiliated Sixth People's Hospital, 600 Yishan Road, Shanghai 200233, P.R. China

E-mail: zzl2002@medmail.com.cn

Key words: osteogenesis imperfecta, Serpin family F member 1, FK506 binding protein 10, mutation, Chinese patients proband displayed a novel compound heterozygous mutation in FKBP10, c.813_814delGA (p.Glu271 AspfsX101) and c.831delC (p.Gly278AlafsX20), and did not have Bruck syndrome. Codon 831 of the FKBP10 gene may represent a mutation hotspot for human OI. These results extend both the phenotypic and the genotypic contents of OI patients with SERPINF1 or FKBP10 mutations.

\section{Introduction}

Osteogenesis imperfecta (OI) is a clinically heterogeneous and heritable connective tissue disorder that is characterized by bone fragility and susceptibility to fractures following minimal trauma (1). Sillence et al (2) initially categorized OI individuals into four types based on clinical features and disease severity: Type I [(Mendelian Inheritance in Man (MIM) 166200], type II (MIM 166210), type III (MIM 259420), and type IV (MIM 166220). For the majority of patients, OI is associated with mutations of COL1A1 (MIM 120150) or COL1A2 (MIM 120160), which encode type I collagens (3). Glorieux et al (4) described a group of patients with OI that presented with a discrete phenotype, including hyperplastic callus formation and calcification of the interosseous membrane; this was designated as OI type V (MIM 610967). In 2002, Glorieux et al (5) described another novel form of OI that presented with osteoid accumulation due to a mineralization defect; this was designated OI type VI (MIM 613982). Patients with OI type VI typically sustain their first fracture after the age of 6 months (6). Their sclerae are white or faintly blue and dentinogenesis imperfecta (DI) is uniformly absent (6). These characteristic features of OI type VI are readily apparent in histological bone samples, including bone biopsy specimens that have a 'fish-scale' pattern, hyperosteoidosis, prolonged mineralization lag time and decreased mineral apposition rate (5). Becker et al (7) first reported that Serpin family F member 1 (SERPINF1) mutations cause severe autosomal recessive OI in 2011. FK506 binding protein 10 (FKBP10) mutations were first reported by Alanay et al (8) in 2010 in autosomal-recessive OI. Kelley et al (9) concluded that FKBP10 mutations also cause Bruck syndrome, which is characterized 
by congenital joint contractures. Autosomal-recessive OI with FKBP10 mutations was designated as OI type XI (10). As an increasing number of virulence genes have been discovered, the OI classification has expanded to 15 types (10).

Previous studies have reported that the most prevalent pathogenic genes responsible for OI in Chinese patients were COL1A1 and COL1A2 $(11,12)$. To the best of our knowledge, only one report of Chinese patients with SERPINF1 mutations and one with FKBP10 mutation have previously been reported, respectively $(13,14)$. In the present study, two novel mutations in SERPINF1 and one novel compound heterozygous mutation in FKBP10 were identified in three unrelated Chinese families with autosomal recessive OI type VI and XI.

\section{Materials and methods}

Subjects. None of the probands belonged to consanguineous families. Three unrelated Chinese families with OI (Fig. 1) and 250 healthy control donors were included in the present study. Patients were recruited between May 2010 and May 2015 from our outpatient clinic, Tianlin Street Community Centre and Fengin Street Community Centre. The control donors included 129 females (66.6 \pm 10.0 years) and 121 males (59.3 \pm 17.9 years). All patients and control subjects belonged to the Han ethnic group.

Ethics statement. The present study was approved by the Ethics Committee of the Shanghai Jiao Tong University Affiliated Sixth People's Hospital (Shanghai, China). All adult participants provided written informed consent prior to beginning the study. In addition, written informed consent was provided by the parents on behalf of the children enrolled in the present study.

Bone densitometry. The bone mineral density (BMD; $\mathrm{g} / \mathrm{cm}^{2}$ ) of the lumbar spine (L1-L4) was measured using dual-energy X-ray absorptiometry (DXA). The probands of families 1 (proband 1) and 3 (proband 3) were assessed using Lunar Prodigy equipment (GE Lunar Corp., Madison, WI, USA). The Lunar device was calibrated daily and the coefficient of variability $(\mathrm{CV})$ value of the DXA measurements at L1-4 was $1.39 \%$ (15). The proband of family 2 (proband 2) was assessed using Hologic Discovery A equipment (Hologic, Bedford, MA, USA). The machine was calibrated daily. The $\mathrm{CV}$ value of the DXA measurements at L1-4 was 0.9\% (15). Lumbar spine $\mathrm{BMD}$ results were converted to age- and sex-specific Z-scores according to the reference data (16). There are 3 DXAs in the department, including 2 GE Lunar equipment and 1 Hologic Discovery A equipment. The patients were examined using the DXAs depending on availability.

Laboratory tests. Fasting blood samples were obtained between 8 and $10 \mathrm{am}$. Blood was stored for $2-3 \mathrm{~h}$ at $25^{\circ} \mathrm{C}$ and centrifuged at $4^{\circ} \mathrm{C}$ for $10 \mathrm{~min}$ at $4,000 \mathrm{x}$ g to obtain the serum. The following markers of calcium metabolism were measured: Serum calcium $(\mathrm{Ca})$, phosphorus $(\mathrm{P})$, alkaline phosphatase (ALP), intact parathyroid hormone (PTH), and 25 hydroxy vitamin D3 [25(OH)D]. Ca, P and ALP were measured using a HITACHI7600-020 automatic biochemistry analyser (Hitachi, Ltd., Tokyo, Japan). All other compounds were measured using the following kits: Intact PTH kit (Roche Diagnostics GmbH, Mannheim, Germany) and a 25(OH)D kit (Roche Diagnostics GmbH, Mannheim, Germany). The intra- and inter-assay CVs, respectively, were 1.5 and $2.0 \%$ for $\mathrm{Ca}, 2.1$ and $2.3 \%$ for P, 2.5 and $4.5 \%$ for ALP, 5.7 and $7.3 \%$ for $25(\mathrm{OH}) \mathrm{D}$ and 1.4 and $2.9 \%$ for PTH as previously reported (17). Pigment-epithelium-derived factor (PEDF) was measured using an ELISA reader (R\&D Systems, Inc., Minneapolis, MN, USA). The intra- and inter-assay coefficients of variation were 8.3 and $9.2 \%$, respectively.

Whole exome capture and massively parallel DNA sequencing. The exome of proband 1 and proband 3 were sequenced to identify the pathogenic gene. Exon-enriched DNA was sequenced using an Illumina Genome Analyser II platform according to the manufacturer's protocol (Illumina, Inc., San Diego, CA, USA). The raw image files were processed using Illumina Base Calling Software v. 1.7 with default parameters and the sequence of each individual DNA fragment was reported as 101-bp paired-end reads. The sequencing reads were aligned to the NCBI human reference genome (NCBI36.3; https://www.ncbi.nlm.nih.gov/assembly/GCF_000001405.12/) using SOAPaligner 2.21 (18-20). The SOAPsnp results were filtered using the following standards: The base quality was $\geq 20$, the sequencing depth was between 50-200 and the distance between two single-nucleotide polymorphisms (SNPs) was $>5$ bp (21-23). Approximately 114 million reads were quantified and mapped to the hs37d5 human reference genome DNA sequence, giving an average read depth of 70.0-96.5 for the exome of each proband. The percentage of the official target covered with at least 20X was $89.06 \%$. All 1000G and $\mathrm{dbSNP}$ records, the filter region (including exonic or splicing) and the overlapping non-coding RNA regions were filtered out. Approximately $11.4 \mathrm{~Gb}$ of high-quality data were aligned to the target regions of the proband with a per-base mismatch rate of $0.52 \%$. As a result, the mean coverage sequencing depth on the official target was 79. Reads that were among the target regions for SNP identification were collected for subsequent analysis. The consensus sequence and quality of each allele were calculated by SOAPsnp.

Sanger sequencing analysis. As whole-exome sequencing is expensive, Sanger sequencing of SERPINF1 was used for other OI probands without COL1A1 and COL1A2 mutations. Mutations in SERPINF1 for proband 2 were assessed. The results of exome sequencing for families 1 and 2 were verified using Sanger sequencing analysis of the SERPINF1 gene. All eight exons and the exon-intron boundaries of SERPINF1 were amplified from genomic DNA via polymerase chain reaction (PR). Patient sequences were referenced to the Ensembl gene sequence ENSG00000132386 (SERPINF1; https://www.ncbi .nlm.nih.gov/nuccore/NC_000017.11?from $=1761965 \&$ to $=1777$ $565 \&$ report=genbank).

The results of next-generation Sanger sequencing analysis of the FKBP10 gene were used to verify Family 3. All eleven exons and the exon-intron boundaries of FKBP10 were amplified from genomic DNA via PCR. Patient sequences were referenced to the Ensembl gene sequence ENSG00000141756 (FKBP10; https://www.ncbi.nlm.nih.gov/nuccore/NC_000017 .11 ?from $=41812262 \&$ to $=41823217 \&$ report=genbank). 
Direct sequencing was performed using the BigDye Terminator Cycle Sequencing Ready Reaction kit, v. 3.1 (Applied Biosystems; Thermo Fisher Scientific, Inc., Waltham, MA, USA), and the sequencing was analysed with an ABI 3730XL automated sequencer (Applied Biosystems; Thermo Fisher Scientific, Inc.). SNPs were identified using Polyphred (http://droog.gs.washington.edu/polyphred). Furthermore, Protein Variation Effect Analyzer (PROVEAN; http://provean .jcvi.org/) and Polymorphism Phenotyping (POLYPHEN-2; http://genetics.bwh.harvard.edu/pph2/) tools were used to predict whether an amino acid substitution had an impact on the biological function of the protein.

Quantitative PCR (qPCR) and detection of the allelic copy numbers of the two families to verify deletion. The following primers were used in the present study and were designed using Primer 3 software (http://frodo.wi.mit.edu/ cgi-bin/primer3/primer3_www.cgi): Primers corresponding to the head site of the targeted gene SERPINF1, forward 5'-GCCTGCTGGACGCTGGATTA-3' and reverse 5'-CAC CCAGCCTAGTCCCTCTAAGC-3'; primers corresponding to the mutagenic site of proband 2 on the targeted gene SERPINF1, forward 5'-CCATCATTCACCGGGCTCTCT-3' and reverse 5'-CGGGAGGCACTCTTGAGGTTC-3'; primers corresponding to the tail site of the targeted gene SERPINFI forward, 5'-TGGCTTTGAGTGGAACGAGGA-3' and reverse 5'-TGATAGTCCAGCGGAAGGTG-3'. The mutagenic site of proband 1 was near the tail of the gene region; as such, family 1 only needed two pairs of primers corresponding to the head and tail regions. However, family 2 required two pairs of primers corresponding to the head, tail and mutated regions. All DNA samples from the 2 families were detected using 2X SYBR Quant Master Mix (Applied Biosystems; Thermo Fisher Scientific, Inc.) qPCR assays and the reference gene was RPP14. Thermo cycling conditions were as follows: $95^{\circ} \mathrm{C}$ for $2 \mathrm{~min}$ followed by 40 cycles of $95^{\circ} \mathrm{C}$ for $15 \mathrm{sec}$ and $60^{\circ} \mathrm{C}$ for $15 \mathrm{sec}$ followed by the dissociation step. All reactions were performed in triplicate. The allelic copy number of the markers in the muted region was finally determined with the comparison of normalized data of control samples and patients using the $2^{-\Delta \Delta \mathrm{Cq}}$ method (24).

\section{Results}

Clinical features Family 1. Proband 1 was an 11-year-old girl, the first daughter of non-consanguineous parents. She was the product of a full-term pregnancy with a normal delivery (Fig. 1), and her birth weight and length were within normal limits. Her height and weight at the time of the study were $132.6 \mathrm{~cm}$ (Z score, -1.7) and $35.4 \mathrm{~kg}$ (Z score, -0.3), respectively. She experienced her first fracture in the femur at the age of 2 years and has since experienced 5 subsequent fractures, including both femurs between 8 and 11 years. Proband 1 has evident deformities in both lower limbs. Blue sclera, brittle teeth and hearing loss were not observed and no family history of bone fragility was identified. Radiographs revealed thin cortices and normal diaphyseal modelling with bending deformities in both femurs (Fig. 2A). Radiographs of the thoracic vertebra and lumbar vertebra of proband 1 revealed multiple vertebral wedging, she did not feel any pain (Fig. 2B). Proband 1 was

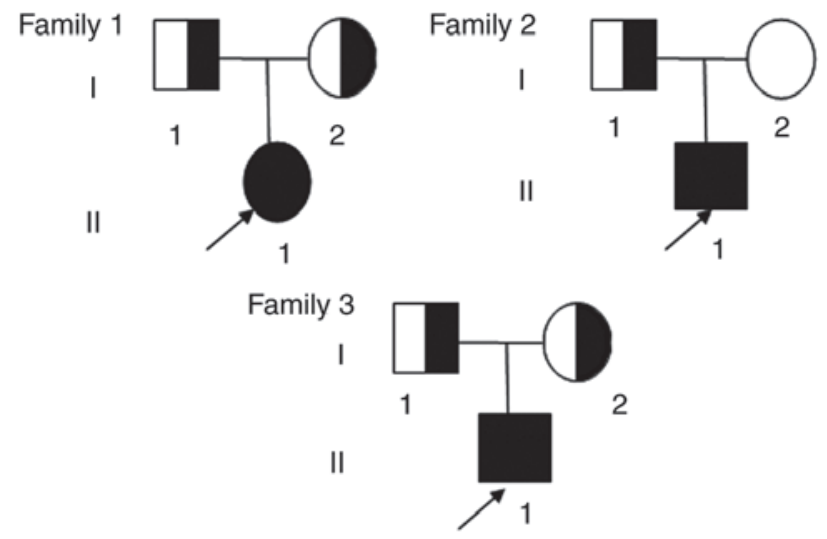

Figure 1. Pedigrees of the families included in the present study. Arrows indicate probands. Shaded symbols represent patients with osteogenesis imperfecta, half shaded symbols represent asymptomatoc carriers. Squares represent males and circles represent females.

demonstrated to have a lower height and lumbar spine bone mass compared with healthy donors of the same age (Table I). Biological test results, including serum Ca, P, ALP and PTH were within normal ranges, whereas $25(\mathrm{OH}) \mathrm{D}$ levels were below the normal range (Table I). Her parents did not show any symptom of OI, except that her father was below average height (159.9 cm; Z score, -1.9$)$.

Family 2. Proband 2 was a 4-year-old boy, the only son of non-consanguineous parents (Fig. 1). He was the product of a full-term pregnancy with a normal delivery, and his birth weight and length were within normal limits. His height and weight at the time of the study were $102.4 \mathrm{~cm}$ ( $\mathrm{Z}$ score, -0.3$)$ and $16.1 \mathrm{~kg}$ (Z score, -0.1), respectively. He experienced his first fracture in the left upper femur at the age of 3 years. Subsequently, he experienced another fracture in the left middle femur at the age of 4 years. Proband 2 presented with blue sclera without brittle teeth or hearing loss. Radiographs revealed thin cortices, normal diaphyseal modelling in the left femur and multiple vertebral wedging (Fig. 2C and D). Proband 2 had a decreased lumbar spine bone mass compared with healthy controls of the same age. All biological test results were within normal ranges (Table I). His parents did not exhibit any symptoms of OI.

Family 3. Proband 3 was a 12 -year-old boy, the only son of non-consanguineous parents (Fig. 1). He was the product of a full-term pregnancy with a normal delivery, and his birth weight and length were within normal limits. His height and weight at the time of the study were $140.0 \mathrm{~cm}(\mathrm{Z}$ score, -1.9$)$ and $34.0 \mathrm{~kg}$ ( $\mathrm{Z}$ score, -0.7$)$, respectively. He experienced his first fracture in the right upper femur at the age of 10 years, with 4 subsequent fractures of the right femur. Proband 3 was unable to walk without the aid of auxiliary equipment following the first fracture. He did not present with blue sclera, brittle teeth, hearing loss or congenital large joint contracture. No family history of bone fragility was identified. Radiographs revealed thin cortices, normal diaphyseal modelling and bending deformities in the right femur, whilst a chest X-ray identified light scoliosis (Fig. 2E and F). Proband 3 had a lower lumbar spine bone mass compared with healthy controls of the 
A

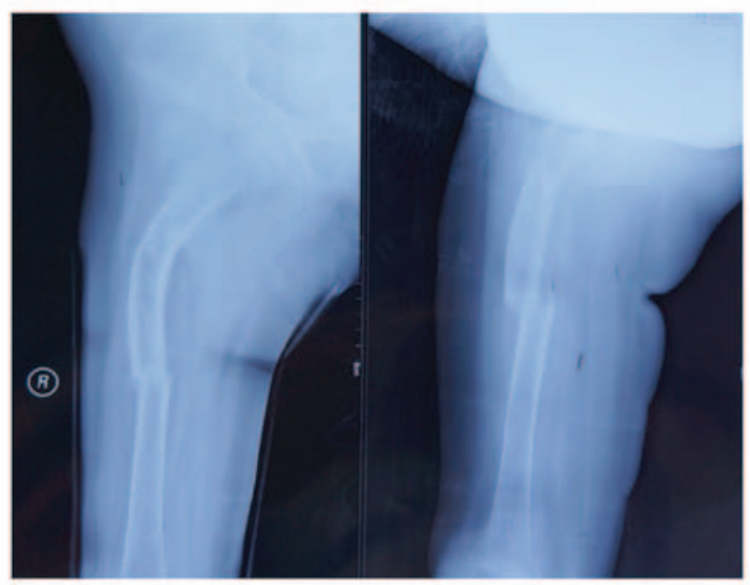

C

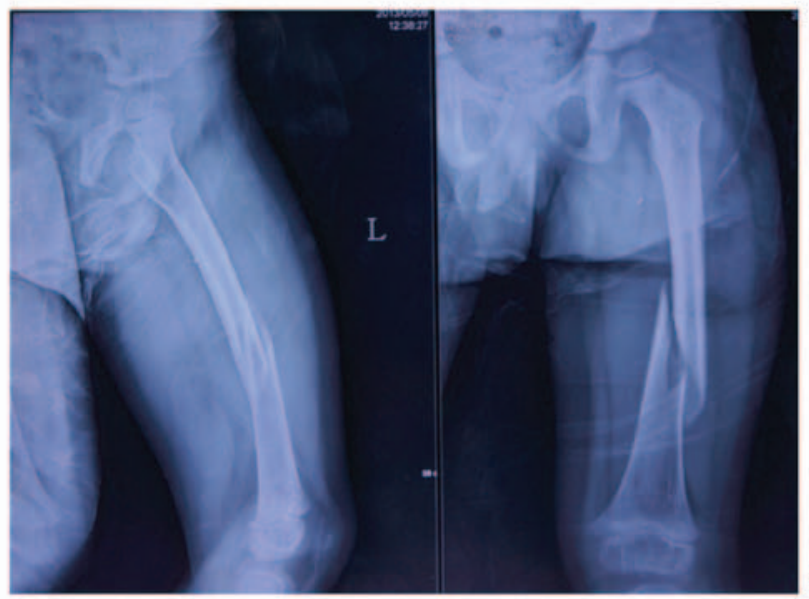

$\mathrm{D}$

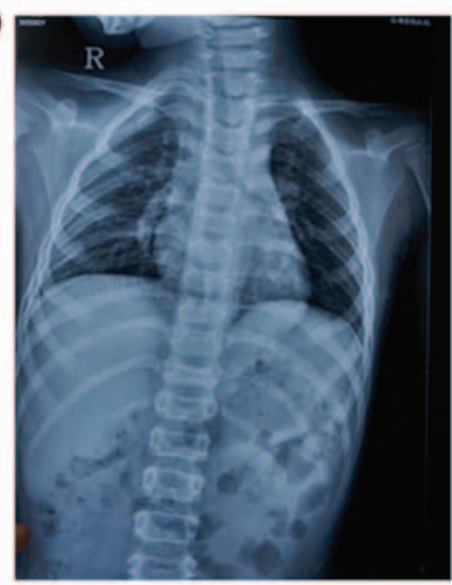

$\mathrm{B}$

E
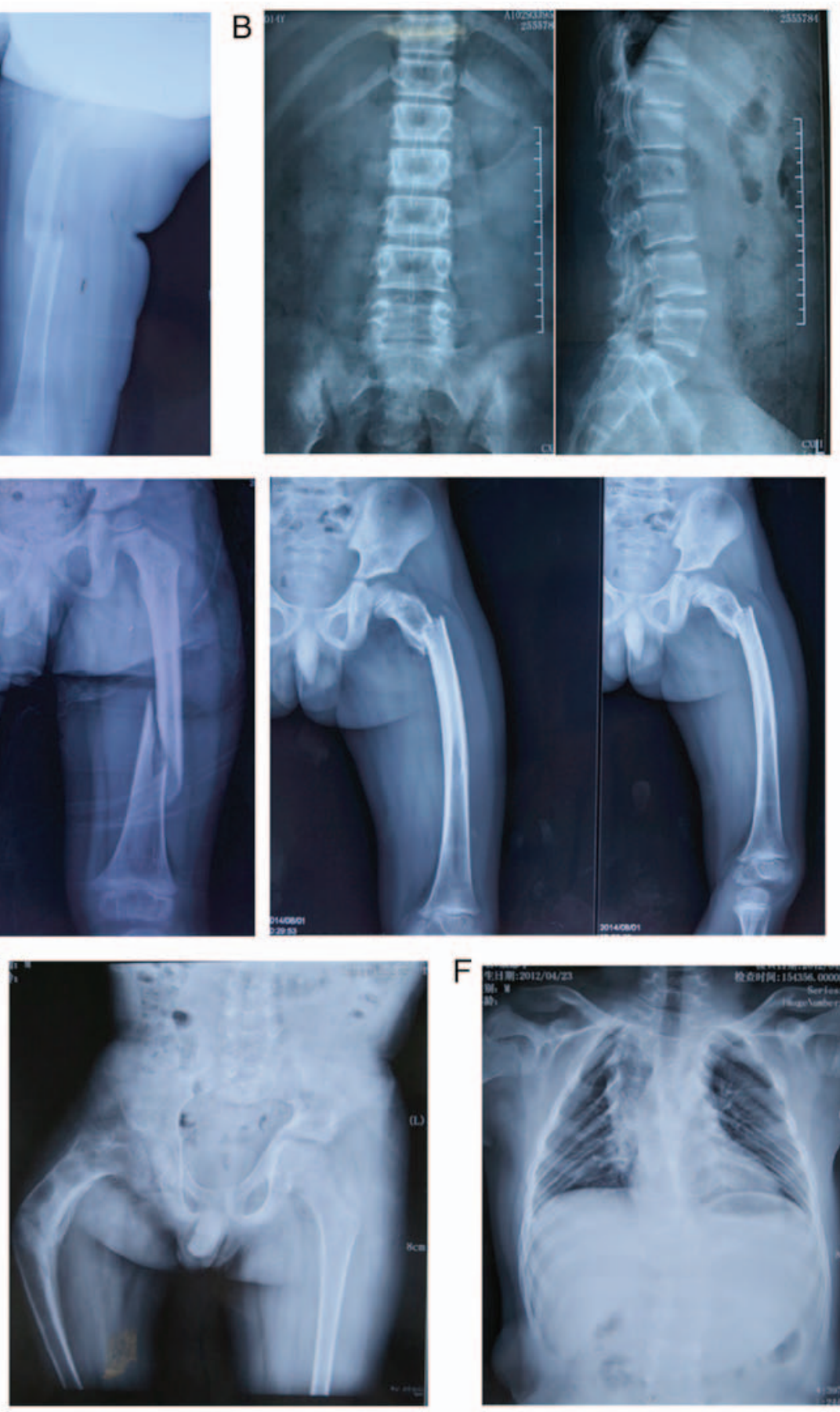

$\mathrm{F}$

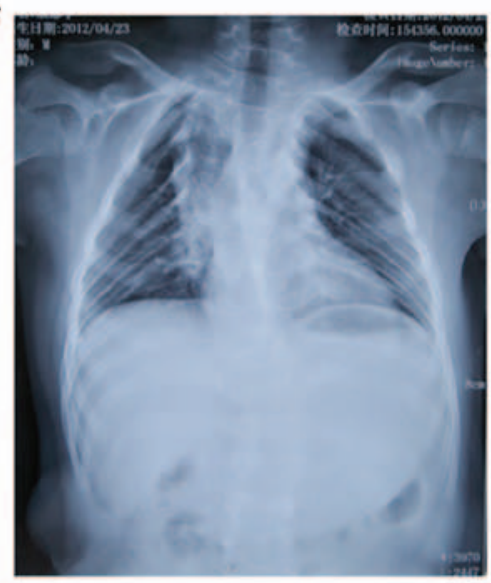

Figure 2. Radiographs of disease manifestations in three probands. (A) Anterioposterior and lateral images of the right leg of proband 1 with severe bowing of the right femur and multiple old and new fractures. (B) Radiographs of the thoracic and lumbar vertebrae of proband 1 with multiple vertebral wedging. (C) Radiographs of the left leg of proband 2, with two fractures and bowing of the left femur. (D) Chest radiograph of proband 2 revealed multiple vertebral wedging of thoracic vertebrae 11 and 12 and lumbar vertebra 1 with no signs of rib or clavicular fractures. (E) Radiographs of the right leg of proband 3 revealed severe bowing and multiple fractures of the right femur. (F) Chest radiograph of proband 3 revealed light scoliosis.

same age. Biological test results, including serum Ca, P, ALP and PTH, were within normal ranges. However, proband 3 was identified to have a $25(\mathrm{OH}) \mathrm{D}$ deficiency (Table I). His parents did not show any other symptoms.

Whole exome capture and massively parallel DNA sequencing. A novel missense substitution was identified in the SERPINF1 gene of proband 1. This mutation was homozygous according to the autosomal recessive mode of inheritance.
The homozygous $\mathrm{T}$ to A transition at c.1067 in exon 8 of SERPINF1 (Fig. 3) resulted in a valine to glutamic acid substitution at p.356 (V356E). This homozygous missense mutation occurred at a highly conserved position (Fig. 4). The results revealed that this missense mutation was deleterious with a PROVEAN score of -4.334. A similar result was obtained when using POLYPHEN-2.

A novel compound heterozygous mutation that consisted of a nonsense mutation (c.813_814delGA, p.Glu271AspfsX101) 
Table I. General features and laboratory findings of the probands.

\begin{tabular}{|c|c|c|c|c|}
\hline Parameter & Proband 1 & Proband 2 & Proband 3 & Normal values \\
\hline Sex & $\mathrm{F}$ & M & M & \\
\hline Age (years) & 11 & 4 & 12 & \\
\hline Blue sclera & - & + & - & \\
\hline Dentinogenesis imperfecta & - & - & - & \\
\hline Hearing loss & - & - & - & \\
\hline Site of fracture & $\mathrm{B}$ & $\mathrm{L}$ & $\mathrm{R}$ & \\
\hline First fracture age (years) & 2 & 3 & 10 & \\
\hline Times of fracture & 6 & 2 & 5 & \\
\hline Height Z-score & $-1.7^{\mathrm{a}}$ & -0.3 & $-1.9^{a}$ & $>-1$ \\
\hline Weight Z-score & -0.3 & -0.1 & -0.7 & $>-1$ \\
\hline Lumbar spine 1-4 Z-score & $-2.9^{\mathrm{a}}$ & $-2.6^{\mathrm{a}}$ & $-5.9^{\mathrm{a}}$ & $>-1$ \\
\hline Calcium (mmol/l) & 2.42 & 2.49 & 2.40 & $2.08-2.60$ \\
\hline Phosphate (mmol/l) & 1.39 & 1.27 & 1.02 & $0.80-1.60$ \\
\hline Alkaline phosphate (U/)1 & 224 & 365 & 350 & $116-380$ \\
\hline Parathyroid hormone (pg/ml) & 58.0 & 29.3 & 54 & $15.0-65.0$ \\
\hline $25(\mathrm{OH}) \mathrm{D}(\mathrm{ng} / \mathrm{ml})$ & $16^{\mathrm{a}}$ & 34 & $18^{\mathrm{a}}$ & $>30$ \\
\hline
\end{tabular}

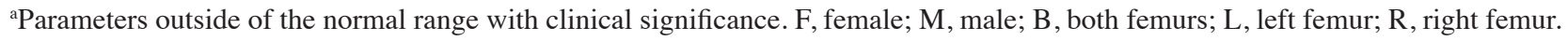

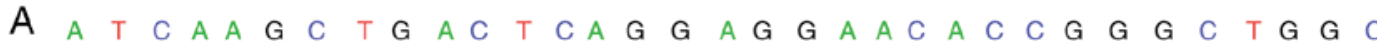

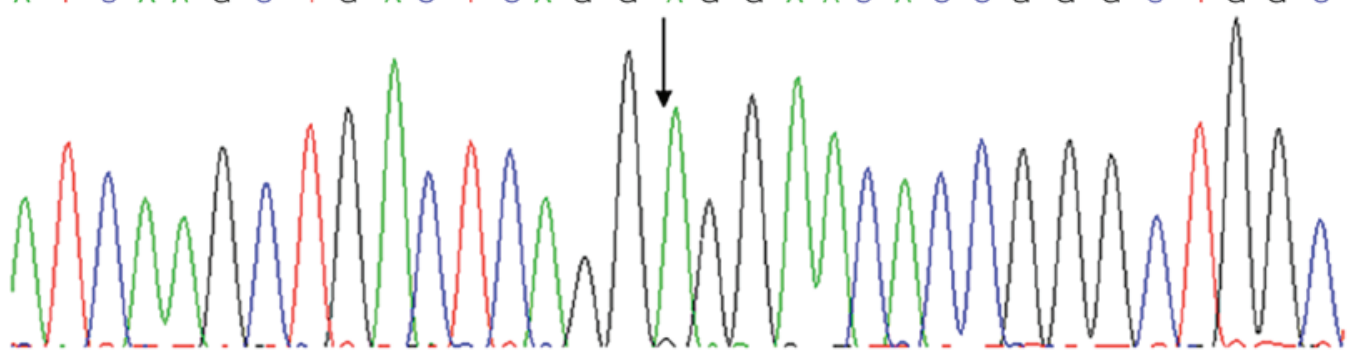

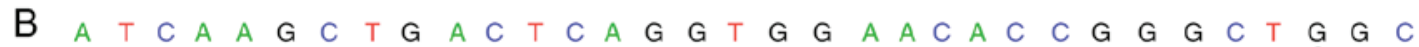

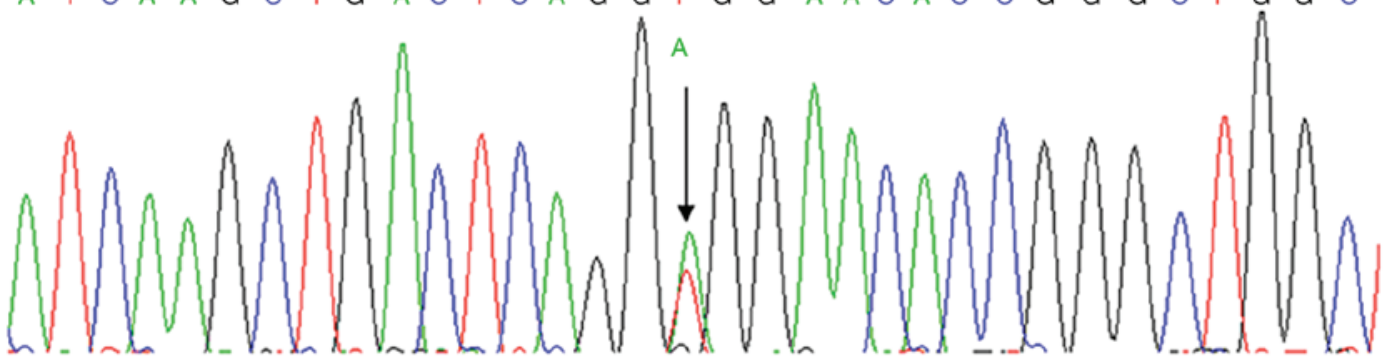

C C C T A A C T G G A G T CA G TGG TGCGATCTCA G C T C A A T G C A A C CA A G G C T G A A T G C A T G G T G T

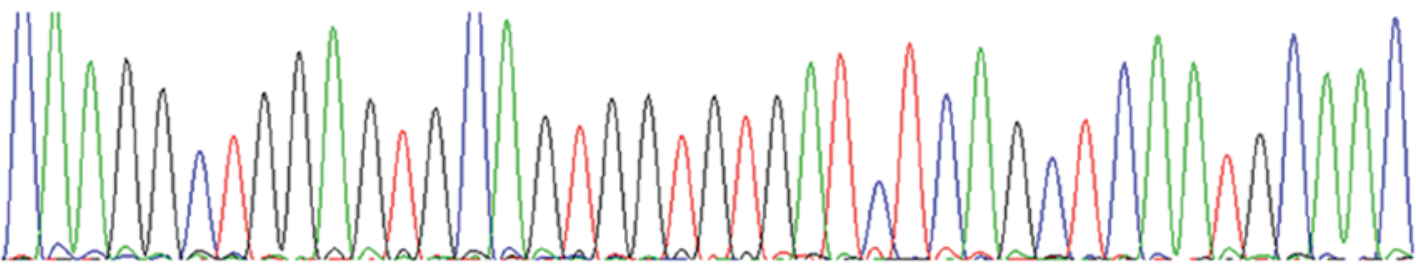

Figure 3. Genetic analysis of the Serpin family F member 1 gene mutation in family 1 and family 2. (A) Direct DNA sequencing of proband 1 . Arrow indicates the homozygous missense mutation c.1067T >A (V356E). (B) Mutation screening was performed in the father of proband 1, a heterozygous carrier. Arrow indicates the site of the heterozygous missense mutation c.1067T >A (V356E). (C) Direct DNA sequencing of proband 2 and his father. The proband had homozygous deletion of a 1,657 bp base sequence including exon 4, intron 4, exon 5 and parts of intron 3 and 5, c.283+473_643+104del (p.Ala96_Gly215del). The DNA sequencing chart identified the chain with deletions in the proband's father, who had heterozygous deletion. 


$\begin{array}{lc} & 338 \\ \text { Human } & \text { FDSPDFSKITGKPIKLTQVE } \\ \text { Rat } & \text { FESPDFSKITGKPVKLTQVE } \\ \text { Mouse } & \text { FESPDFSKITGKPVKLTQVE } \\ \text { Cattle } & \text { FDAPDFSKITGKPIKLTQVE } \\ \text { Sheep } & \text { FDAPDFSKITGKPIKLTQVE } \\ \text { Western clawed frog } & \text { FATPDFSKISSKPLKLSYVV } \\ \text { Zebrafish } & \text { FDSPDFSKITGKPIKLTQVE } \\ \text { Dog } & \text { FDSPDFSKITGKPIKLTQVE } \\ \text { Cynomolgus monkey } & \text { FDSPDLSKITGKAIKLTQVE } \\ \text { Sarcophilus harrisii } & \end{array}$

Figure 4. Homologous sequences of SERPINF1 across species. The missense mutation p.Val356Glu observed in proband 1 occurs at a highly conserved position in SERPINF1. The bases that are identical to those in Homo sapiens are displayed in the box. SERPINF1, Serpin family F member 1.

and another nonsense mutation (c.831delC,p.Gly278AlafsX20) was identified in exon 5 of the FKBP10 gene in proband 3 (Fig. 5A and B).

Validation of the SERPINF1 and FKBPIO germline mutation. The Sanger sequencing results for the SERPINF1 gene were consistent with the whole exome sequencing results. A homozygous V356E (c.1067T >A) mutation was identified in proband 1. The heterozygous V356E (c.1067T >A) mutation was found in proband 1's father (Fig. 3B). However, no mutation in SERPINF1 was observed in the proband's mother, and no V356E mutation was identified in the control subjects. According to OI variant databases (https://oi.gene.le.ac.uk/
variants.php?select_db=SERPINF1\&action=view_all), the identified mutation is novel.

A homozygous p.Ala96_Gly215del (c.283+473_643+ 104del) mutation of SERPINF1 was identified in proband 2 (Fig. 3C). The same heterozygous mutation was identified in the proband's father, whereas no mutation was observed in the proband's mother. The same compound heterozygous mutation was identified in proband 3 by further Sanger sequencing. The heterozygous mutation (c.831delC, p.Gly278AlafsX20) of FKBP10 was present in proband 3's father, whereas the heterozygous mutation (c.813_814delGA, p.Glu271AspfsX101) was identified in the proband's mother.

In the present study, qPCR was repeatedly performed to detect allelic copy numbers of both families and verify whether there were deletions. The results for family 1 revealed a deletion of SERPINF1 in the proband and her mother. However, the results for family 2 demonstrated that there was no SERPINF1 deletion in the proband or her mother. Furthermore, the genotypes of all SNPs of proband 2 were homozygous. This suggests that, due to the uniparental disomy, the region of the chromosome of the proband of family 2 was derived from the father although the total copy number was unchanged.

Detection of serum PEDF. PEDF was undetectable in serum samples from proband 1. Serum samples were not obtained from her parents as initially only the whole blood of the parents was reserved and they did not return to have the serum extracted. Serum PEDF was also undetectable in proband 2. However, for proband 2's father and mother, the PEDF serum concentration was 13.8 and $37.8 \mu \mathrm{g} / \mathrm{ml}$, respectively. PEDF serum concentrations were determined for 10 normal subjects. The median value was $22.4 \mu \mathrm{g} / \mathrm{ml}$, the minimum was $12.0 \mu \mathrm{g} / \mathrm{ml}$ and the maximum was $39.5 \mu \mathrm{g} / \mathrm{ml}$.

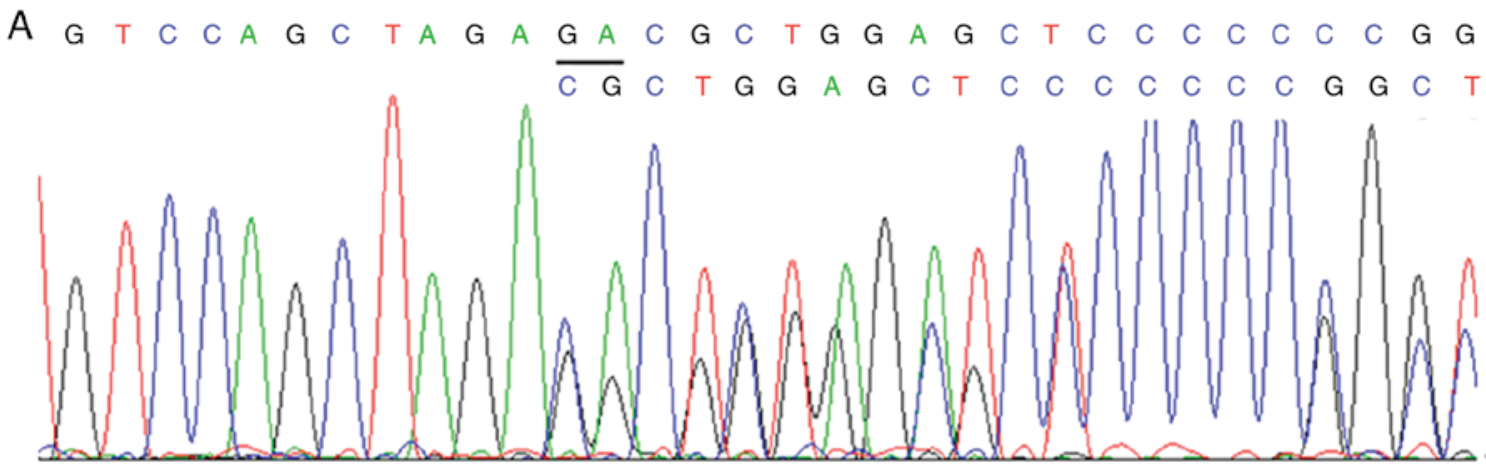

B

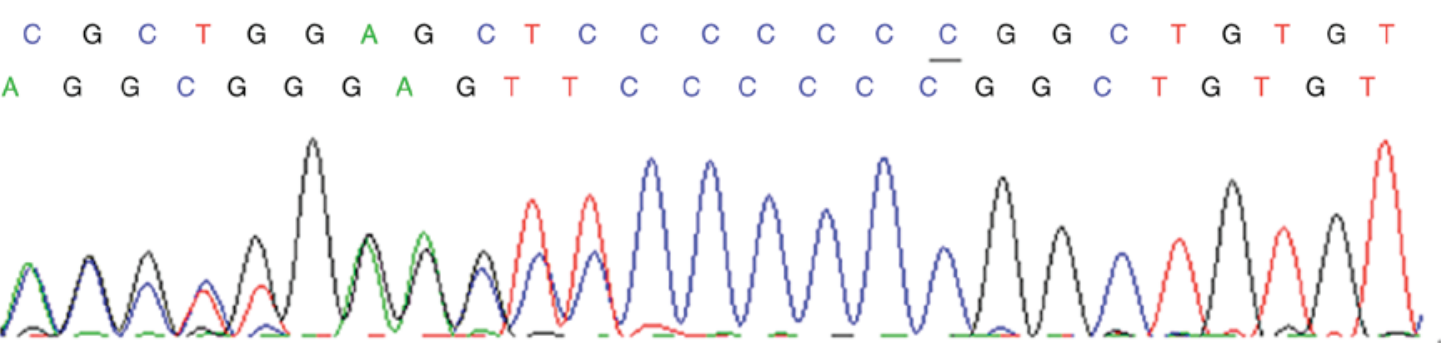

Figure 5. Direct DNA sequencing of proband 3. The proband exhibited a compound heterozygous mutation in FK506 binding protein 10 (A) c.813_814delGA, (p.Glu271AspfsX101) deletion mutation in exon 5 (B) and the other deletion mutation in exon 5 c.831delC(p.Gly278AlafsX20). 


\section{Discussion}

The first causal gene of recessive OI, CRTAP (MIM 605497), was reported in $2006(25,26)$. A number of additional genes, including LEPRE1 (MIM 610339), PPIB (MIM 123841), FKBPIO (MIM 607063), SERPINH1 (MIM 600943), and SP7 (MIM 606633) mutations, have since been reported to cause severe or lethal autosomal recessive OI $(8,27-30)$. Becker et al (7) identified a lack of homozygosity of the SERPINF1 gene product, PEDF, which caused severe OI in four patients. Alanay et al (8) first discovered homozygosity for mutations in FKBP10 in a cohort of five consanguineous Turkish families and a Mexican-American family, causing autosomal-recessive OI.

The SERPINF1 gene is located on chromosome 17p13.3 and encodes PEDF, which is a glycoprotein of the Serpin superfamily secreted by retinal pigment epithelial cells (7). In the musculoskeletal system, collagen-1 is bound by PEDF, which may act as an inhibitor of bone resorption by inhibiting osteoclast maturation via osteoprotegerin and receptor activator of nuclear factor-kB ligand (7). Li et al (31) observed that PEDF suppressed the expression of genes that inhibit mineralization, leading to enhanced osteoblastic differentiation and increased matrix mineralization. In the present study, probands 1 and 2 experienced their first fracture when they were older than 1 year. Both probands suffered recurrent fractures of the femur, with proband 1 more severely affected than proband 2. Proband 1 was 11 years old and proband 2 was 4 years old at the time of the present study; this discrepancy in age may be responsible for the difference in phenotypic severity of OI. The sclera of proband 1 was white, whereas that of proband 2 was faintly blue. Both probands lacked dentinogenesis imperfecta. The phenotype of Chinese patients in the present study was similar to that of other patients reported in previous studies $(5,7,13,32-35)$. Both probands were able to walk independently during the intermittent period between fractures, although they fractured their femurs many times. However, Caucasian patients with OI typically experience severe limb deformity and are mostly unable to walk independently $(5,7,32-35)$. Phenotypes of Korean patients with type VI OI and Inuit patients have also been reported to be more severe compared with those presented in this study $(5,35)$. Probands 1 and 2 presented with multiple vertebral wedging, however they did not report experiencing backache or lumbago. Moderate to severe bone fragility was observed in Chinese patients by Wang et al (13), further supporting the hypothesis that the phenotype of Chinese patients with OI is less severe compared with Caucasian and Korean patients.

To date, a total of 82 mutations in the SERPINF1 gene, including 37 substitutions, 23 deletions, 20 duplications and 2 insertions, have been described (https://oi.gene.le.ac.uk/variants.php?select_db=SERPINF1\&action=view_unique). The novel missense mutation identified in proband 1 consisted of a homozygous $\mathrm{T}$ to A transition at c.1067 in exon 8 (V356E) of SERPINF1. In proband 2, the novel deletion mutation identified comprised a homozygous p.Ala96_Gly215del (c.283+473_643+104del) deletion of SERPINF1, including the loss of intron 4, exon 4, exon 5 and part of introns 3 and 5 . At present, this mutation is the only large deletion identified that has been identified among mutations of the SERPINF1 gene. The father of proband 2 was demonstrated to have a heterozygous deletion of SERPINF1 with no symptoms of OI and normal stature. This indicates that carriers are asymptomatic despite the large fragment deletion. Proband 2 had comparatively mild symptoms; he was able to walk, jump and play with other children during the intermittent period between fractures. Furthermore, his height is within the normal range. The association between the severe mutation type and relatively mild phenotype of this proband should be further studied. Rauch et al (36) reported that the absence of circulating PEDF was specific to OI type VI. In the present study, probands 1 and 2 had undetectable serum concentrations of PEDF, which indicates that their mutations were pathogenic. In addition, the mutations occurred at a highly conserved position and were predicted as deleterious by PROVEAN and polyphen software.

Interestingly, the families of probands 1 and 2 in the present study exhibited nonpaternity. Both probands had homozygous mutations in the SERPINF1 gene, whilst the fathers from both families showed heterozygous mutations. However, both mothers had no mutation on this allele. The mothers may therefore have a deleted SERPINF1 allele. The results of allelic copy number detection revealed that proband 1 and her mother exhibited a SERPINF1 deletion, meaning that family 1 did not deviate from Mendelian inheritance. However, no deletion of SERPINF1 was observed in family 2. Furthermore, the genotypes of all SNPs of proband 2 were homozygous. It was therefore assumed that the uniparental disomy of proband 2 was in part because his chromosome originated from his father, although the total copy number was unchanged. Wang et al (13) also reported this phenomenon in a family that included a proband with a homozygous in-frame duplication, a heterozygous carrier mother and a father without mutations. It was suggested that Sanger sequencing of this phenomenon may reveal nonpaternity or partial de novo mutation.

The first FKBP10 mutations were identified in families with a moderate to severe form of OI without contractures or webbing (8). Steinlein et al (37) also described patients with FKBP10 mutations affected by severe autosomal recessive OI without contractures. However, Shaheen et al (38) discovered two brothers with Bruck syndrome, in which multiple joint contracture was caused by a mutation in the FKBP10 gene. Kelley et al (9) concluded that FKBPIO mutations are the cause of recessive OI and Bruck syndrome. Zhou et al (14) also reported aChinese patient with OI and Bruck syndrome resulting from mutations in the FKBPIO gene. In the present study, the patients presented with moderate OI without contractures or webbing. The majority of patients with OI experience their first fractures soon after birth or in infancy $(8,14,37)$. However, in the present study, proband 3 suffered his first fracture when he was 10 years old, consistent with the phenotype described by Schwarze et al (39). Proband 3 did not have blue sclerae or dentinogenesis imperfecta and presented with scoliosis that developed during adolescence. His fracture was restricted on the right femur and resulted in deformity in this area. The results of the present study verified that FKBP10 may cause Bruck syndrome or isolated OI. In addition, Schwarze et al (39) reported that isolated OI and Bruck syndrome may occur in siblings of the same family. 
A total of 119 variants in the FKBP10 gene have been identified, including 32 substitutions, 17 deletions, 62 duplications and 8 insertion/deletions and mutations primarily located in exon 5 (32.04\%) and exon 6 (22.33\%) (https://oi.gene.le.ac. uk/variants_statistics.php). The novel compound heterozygous mutation in proband 3 described herein was located in exon 5. Of these mutations, c.831dupC and c.948dupT occur most frequently, having been reported 21 and 19 times, respectively (https://oi.gene.le.ac.uk/variants.php?select_ $\mathrm{db}=$ FKBP10\&action=view_unique). In particular, the mutation c.831dupC has been reported in different origins, including the families of Turkish, Mexican, South African and Caucasian origin $(8,9,39)$. In the present study, proband 3 also had a nonsense mutation located at c.831, with a deleted $\mathrm{C}$ instead of a duplicate $C$. These results suggest that codon 831 of the FKBP10 gene may represent a mutation hotspot for human OI.

In conclusion, the results of the present study indicate that a novel missense mutation, c.1067T $>\mathrm{A}$, in exon 8 and a large fragment deletion mutation, c.283+473_643+104del, in the SERPINF1 gene cause type VI autosomal recessive OI in Chinese patients. The present study also demonstrated that a patient may experience mild symptoms of OI despite having a large fragment deletion in the SERPINFI gene. Furthermore, the phenotype of Chinese patients with type VI OI appears to be less severe compared with Caucasian and Korean patients. Deletion of the SERPINFI gene, as observed in one of the proband's parents, or the uniparental disomy of the proband may result from one heterozygous parent carrier and one parent without identified mutation producing a child with a homozygous mutation. The novel compound heterozygous mutations c.831delC and c.813_814delGA in FKBP10 are responsible for moderate OI without Bruck syndrome in Chinese patients. Codon 831 of the FKBP10 gene may represent a mutation hotspot for $\mathrm{OI}$ in humans. The results of the present study may improve the clinical and pathogenic gene spectrum of recessively inherited forms of OI in China.

\section{Acknowledgements}

The authors would like to thank the Center for Genetic \& Genomic Analysis, Genesky Biotechnologies Inc. (Shanghai, China) for their assistance with gene identification.

\section{Funding}

The present study was supported by the National Basic Research Program of China (grant no. 2014CB942903), National Natural Science Foundation of China (NSFC; grant nos. 81370978 and 30800387), and Chongqing City Fundamental And Advanced Research Projects (grant no. CSTC2013jcyjC00009) and the Science and Technology Commission of Shanghai municipality (grant nos. 14JC1405000 and 14ZR1431900).

\section{Availability of data and materials}

The datasets used and/or analyzed during the current study are available from the corresponding author on reasonable request.

\section{Authors' contributions}

ZZ recruited patients, designed the study, revised and approved the final version of the manuscript. HZ recruited patients, performed experiments, and wrote the manuscript. YX, HY and $\mathrm{CW}$ performed experiments and revised the manuscript. $\mathrm{JG}, \mathrm{JH}, \mathrm{WF}$ and $\mathrm{WH}$ performed experiments and analyzed the data. All authors read and approved the final manuscript.

\section{Ethics approval and consent to participate}

The present study was approved by the Ethics Committee of the Shanghai Jiao Tong University Affiliated Sixth People's Hospital (Shanghai, China). All adult participants provided written informed consent prior to beginning the study. In addition, written informed consent was provided by the parents on behalf of the children enrolled in the present study.

\section{Consent for publication}

All the probands and their parents consented to publication of this study.

\section{Competing interests}

The authors declare that they have no competing interests.

\section{References}

1. Rauch F and Glorieux FH: Osteogenesis imperfecta. Lancet 363: 1377-1385, 2004.

2. Sillence DO and Rimoin DL: Classification of osteogenesis imperfect. Lancet 1: 1041-1042, 1978.

3. Cheung MS and Glorieux FH: Osteogenesis imperfecta: Update on presentation and management. Rev Endoc Metab Disord 9: 153-160, 2008.

4. Glorieux FH, Rauch F, Plotkin H, Ward L, Travers R, Roughley P, Lalic L, Glorieux DF, Fassier F and Bishop NJ: Type V osteogenesis imperfecta: A new form of brittle bone disease. J Bone Miner Res 15: 1650-1658, 2000.

5. Glorieux FH, Ward LM, Rauch F, Lalic L, Roughley PJ and Travers R: Osteogenesis imperfecta type VI: A form of brittle bone disease with a mineralization defect. J Bone Miner Res 17: 30-38, 2002.

6. Tucker T, Nelson T, Sirrs S, Roughley P, Glorieux FH, Moffatt P, Schlade-Bartusiak K, Brown L and Rauch F: A co-occurrence of osteogenesis imperfecta type VI and cystinosis. Am J Med Genet A 158A: 1422-1426, 2012.

7. Becker J, Semler O, Gilissen C, Li Y, Bolz HJ, Giunta C, Bergmann C, Rohrbach M, Koerber F, Zimmermann $\mathrm{K}$, et al: Exome sequencing identifies truncating mutations in human SERPINF1 in autosomal-recessive osteogenesis imperfecta. Am J Hum Genet 88: 362-371, 2011.

8. Alanay Y, Avaygan H, Camacho N, Utine GE, Boduroglu K, Aktas D, Alikasifoglu M, Tuncbilek E, Orhan D, Bakar FT, et al: Mutations in the gene encoding the RER protein FKBP65 cause autosomal-recessive osteogenesis imperfecta. Am J Hum Genet 86: 551-559, 2010.

9. Kelley BP, Malfait F, Bonafe L, Baldridge D, Homan E, Symoens S, Willaert A, Elcioglu N, Van Maldergem L, Verellen-Dumoulin $\mathrm{C}$, et al: Mutations in FKBP1O cause recessive osteogenesis imperfecta and Bruck syndrome. J Bone Miner Res 26: 666-672, 2011.

10. Valadares ER, Carneiro TB, Santos PM, Oliveira AC and Zabel B: What is new in genetics and osteogenesis imperfecta classification? J Pediatr 90: 536-541, 2014.

11. Zhang ZL, Zhang H, Ke YH, Yue H, Xiao WJ, Yu JB, Gu JM, $\mathrm{Hu}$ WW, Wang C, He JW and Fu WZ: The identification of novel mutations in COL1A1, COL1A2, and LEPRE1 genes in Chinese patients with osteogenesis imperfecta. J Bone Miner Metab 30: 69-77, 2012. 
12. Zhang H, Yue H, Wang C, Hu W, Gu J, He J, Fu W, Hu Y, Li M and Zhang Z: Clinical characteristics and the identification of novel mutations of COL1A1 and COL1A2 in 61 Chinese patients with osteogenesis imperfecta. Mol Med Rep 14: 4918-4926, 2016.

13. Wang JY, Liu Y, Song LJ, Lv F, Xu XJ, San A, Wang J, Yang HM, Yang ZY, Jiang Y, et al: Novel mutations in SERPINF1 result in rare osteogenesis imperfecta type VI. Calcif Tissue Int 100: 55-66, 2017.

14. Zhou P, Liu Y, Lv F, Nie M, Jiang Y, Wang O, Xia W, Xing X and Li M: Novel mutations in FKBP10 and PLOD2 cause rare Bruck syndrome in Chinese patients. PloS One 9: e107594, 2014.

15. Zhang H, He JW, Gao G, Yue H, Yu JB, Hu WW, Gu JM, Hu YQ, Li M, Fu WZ, et al: Polymorphisms in the HOXD4 gene are not associated with peak bone mineral density in Chinese nuclear families. Acta Pharmacol Sin 31: 977-983, 2010.

16. Maynard LM, Guo SS, Chumlea WC, Roche AF, Wisemandle WA Zeller CM, Towne B and Siervogel RM: Total-body and regional bone mineral content and areal bone mineral density in children aged 8-18 y: The Fels Longitudinal study. Am J Clin Nutr 68 1111-1117, 1998.

17. Lu HK, Zhang Z, Ke YH, He JW, Fu WZ, Zhang CQ and Zhang ZL: High prevalence of vitamin D insufficiency in China: Relationship with the levels of parathyroid hormone and markers of bone turnover. PloS One 7: e47264, 2012.

18. Li R, Li Y, Kristiansen K and Wang J: SOAP: Short oligonucleotide alignment program. Bioinformatics 24: 713-714, 2008.

19. Zhang Z, Xia W, He J, Zhang Z, Ke Y, Yue H, Wang C, Zhang H, $\mathrm{Gu}$ J, Hu W, et al: Exome sequencing identifies SLCO2Al mutations as a cause of primary hypertrophic osteoarthropathy. Am J Hum Genet 90: 125-132, 2012.

20. Li Y, Vinckenbosch N, Tian G, Huerta-Sanchez E, Jiang T, Jiang H, Albrechtsen A, Andersen G, Cao H, Korneliussen T, et al: Resequencing of 200 human exomes identifies an excess of low-frequency non-synonymous coding variants. Nat Genet 42 : 969-972, 2010

21. DePristo MA, Banks E, Poplin R, Garimella KV, Maguire JR, Hartl C, Philippakis AA, del Angel G, Rivas MA, Hanna M, et al: A framework for variation discovery and genotyping using next-generation DNA sequencing data. Nat Genet 43: 491-498, 2011.

22. Wang K, Li M and Hakonarson H: ANNOVAR: Functional annotation of genetic variants from high-throughput sequencing data. Nucleic Acids Res 38: e164, 2010.

23. Shi Y, Li Y, Zhang D, Zhang H, Li Y, Lu F, Liu X, He F, Gong B, Cai L, et al: Exome sequencing identifies ZNF644 mutations in high myopia. PLoS Genet 7: e1002084, 2011

24. Livak KJ and Schmittgen TD: Analysis of relative gene expression data using real-time quantitative PCR and the $2^{-\Delta \Delta C_{\mathrm{T}}}$ method. Methods 25: 402-408, 2001.

25. Morello R,Bertin TK, Chen Y,Hicks J, Tonachini L, Monticone M, Castagnola P, Rauch F, Glorieux FH, Vranka J, et al: CRTAP is required for prolyl 3-hydroxylation and mutations cause recessive osteogenesis imperfecta. Cell 127: 291-304, 2006.

26. Barnes AM, Chang W, Morello R, Cabral WA, Weis M, Eyre DR, Leikin S, Makareeva E, Kuznetsova N, Uveges TE, et al: Deficiency of cartilage-associated protein in recessive lethal osteogenesis imperfecta. N Engl J Med 355: 2757-2764, 2006.

27. Willaert A, Malfait F, Symoens S, Gevaert K, Kayserili H, Megarbane A, Mortier G, Leroy JG, Coucke PJ and De Paepe A: Recessive osteogenesis imperfecta caused by LEPRE1 mutations: Clinical documentation and identification of the splice form responsible for prolyl 3-hydroxylation. J Med Genet 46: 233-241, 2009 .
28. van Dijk FS, Nesbitt IM, Zwikstra EH, Nikkels PG, Piersma SR, Fratantoni SA, Jimenez CR, Huizer M, Morsman AC, Cobben JM, et al: PPIB mutations cause severe osteogenesis imperfecta. Am J Hum Genet 85: 521-527, 2009.

29. Christiansen HE, Schwarze U, Pyott SM, AlSwaid A, Al Balwi M, Alrasheed S, Pepin MG, Weis MA, Eyre DR and Byers PH: Homozygosity for a missense mutation in SERPINH1, which encodes the collagen chaperone protein HSP47, results in severe recessive osteogenesis imperfecta. Am J Hum Genet 86: 389-398, 2010.

30. Lapunzina P, Aglan M, Temtamy S, Caparrós-Martín JA, Valencia M, Letón R, Martínez-Glez V, Elhossini R, Amr K, Vilaboa N and Ruiz-Perez VL: Identification of a frameshift mutation in Osterix in a patient with recessive osteogenesis imperfecta. Am J Hum Genet 87: 110-114, 2010.

31. Li F, Song N, Tombran-Tink J and Niyibizi C: Pigment epithelium derived factor suppresses expression of Sost/Sclerostin by osteocytes: Implication for its role in bone matrix mineralization. J Cell Physiol 230: 1243-1249, 2015.

32. Homan EP, Rauch F, Grafe I, Lietman C, Doll JA, Dawson B, Bertin T, Napierala D, Morello R, Gibbs R, et al: Mutations in SERPINF1 cause osteogenesis imperfecta type VI. J Bone Miner Res 26: 2798-2803, 2011.

33. Venturi G, Gandini A, Monti E, Dalle Carbonare L, Corradi M, Vincenzi M, Valenti MT, Valli M, Pelilli E, Boner A, et al: Lack of expression of SERPINF1, the gene coding for pigment epithelium-derived factor, causes progressively deforming osteogenesis imperfecta with normal type I collagen. J Bone Miner Res 27: 723-728, 2012.

34. Caparrós-Martin JA, Valencia M, Pulido V, Martínez-Glez V, Rueda-Arenas I, Amr K, Farra C, Lapunzina P, Ruiz-Perez VL, Temtamy S and Aglan M: Clinical and molecular analysis in families with autosomal recessive osteogenesis imperfecta identifies mutations in five genes and suggests genotype-phenotype correlations. Am J Med Genet A 161A: 1354-1369, 2013.

35. Cho SY, Ki CS, Sohn YB, Kim SJ, Maeng SH and Jin DK: Osteogenesis imperfecta Type VI with severe bony deformities caused by novel compound heterozygous mutations in SERPINF1. J Korean Med Sci 28: 1107-1110, 2013.

36. Rauch F, Husseini A, Roughley P, Glorieux FH and Moffatt P: Lack of circulating pigment epithelium-derived factor is a marker of osteogenesis imperfecta type VI. J Clin Endocrinol Metab 97: E1550-E1556, 2012.

37. Steinlein OK, Aichinger E, Trucks $\mathrm{H}$ and Sander T: Mutations in $F K B P 10$ can cause a severe form of isolated Osteogenesis imperfecta. BMC Med Genet 12: 152, 2011.

38. Shaheen R, Al-Owain M, Sakati N, Alzayed ZS and Alkuraya FS: FKBP10 and Bruck syndrome: Phenotypic heterogeneity or call for reclassification? Am J Hum Genet 87: 306-307; author reply $308,2010$.

39. Schwarze U, Cundy T, Pyott SM, Christiansen HE, Hegde MR, Bank RA, Pals G, Ankala A, Conneely K, Seaver L, et al: Mutations in FKBP10, which result in Bruck syndrome and recessive forms of osteogenesis imperfecta, inhibit the hydroxylation of telopeptide lysines in bone collagen. Hum Mol Genet 22: $1-17,2013$. 